\title{
A nonlinear control approach for trajectory tracking of slender-body axisymmetric underactuated underwater vehicles
}

\author{
Lam-Hung Nguyen, Minh-Duc Hua, Tarek Hamel
}

\begin{abstract}
This paper develops a novel nonlinear control approach for slender-body underactuated underwater vehicles with a body shape symmetric with respect to the longitudinal axis. Compared to aerial vehicles, added-mass effects are much more preponderant and complex for underwater vehicles, especially for slender bodies. By considering an axisymmetric body, these added-mass effects along with dissipative hydrodynamic force are carefully taken into account via various adaptations and decompositions, resulting in a modified "apparent" force independent of the vehicle's orientation and subsequently a nonlinear system with a triangular control structure. The proposed controller is then complemented with an integral correction term so as to enhance its robustness with respect to model uncertainties and external disturbances. Comparative simulation results conducted on a realistic model of a quasiaxisymmetric underwater vehicle illustrate the performance and robustness of the proposed control approach.
\end{abstract}

\section{INTRODUCTION}

Introduced by Bessel in 1828, the concept of added mass has become widely accepted nowadays. Mostly neglected in modeling and control design of heavier-than-air aircraft, added mass in contrast has always been at the heart of preoccupations of the Underwater Robotics and Automatic Control community [3], [8]. For underwater vehicles, added-mass effects often result in strongly nonlinear dynamics as the total mass matrix can no longer be considered as proportional to identity, except for spherical bodies [3]. These effects are all the more pronounced for underwater vehicles with a slender body shape conceived for reducing hydrodynamic drag along a nominal axis. However, added mass is not the unique source of complexity for control design. The complexity of hydrodynamic effects often impedes obtaining a precise dynamic model, valid in a large operating domain [7]. The vehicles are often subject to strong perturbations (due to currents) whose magnitude can be commensurable with the available actuation power. Therefore, robust nonlinear control design for underwater vehicles is highly recommended.

Control design for underactuated Autonomous Underwater Vehicles (AUV) has received increasing interest during the last three decades [2], [3], [11], [12]. When the control objective concerns the tracking of a reference position trajectory, whose velocity does not vanish for all time, various classical control design methodologies have been applied to provide solutions. For instance, various standard linear and nonlinear control methods have been applied on the

L.-H. Nguyen, M.-D. Hua, and T. Hamel are with I3S, Université Côte d'Azur, CNRS, Sophia Antipolis, France. Prof. Hamel is also with IUF (Institut Universitaire de France), France. Emails: lhnguyen(hua,thamel)@i3s.unice.fr basis of linear approximations of the two subsystems "depthpitch" and "plane-yaw" about nominal operating points (see [3] and the reference therein). The main limitation of these approaches is the local nature of the control design and analysis. Moreover, stability and performance can suffer significantly when strong sea currents or aggressive manoeuvres excite the complex hydrodynamic and added-mass effects. Nonlinear Lyapunov-based control designs have been recently investigated to overcome some of these limitations. Most of them, however, only address the trajectory tracking problem in a horizontal plane, using a simplified and reduced "plane-yaw" 3-DOF model [2], [12]. Few works address this problem in the 3-dimensional space [1], [11]. A nonlinear high-gain backstepping-based controller proposed in [1] allows for exponential convergence of the position error to a small neighborhood of the origin, which means that asymptotic stabilization to zero is not fulfilled. Moreover, the attitude is not explicitly controlled but guided by the closed-loop system's zero dynamics, thus possibly resulting in undesirable attitude dynamics. High-gain controllers are also known to be sensitive to measurement noise and timedelays of control inputs. Refnes et al. [11] have addressed the trajectory tracking for a slender-body underactuated AUV, with a model-based control approach. However, this approach heavily relies on the precision of the model parameters. Control performance can be drastically degraded when model errors are important (see, e.g., [13]). Nonlinear robust control design for underactuated AUVs thus remains an active research topic.

This paper addresses the trajectory tracking control design for slender-body underactuated AUVs, whose body shape is symmetric with respect to the longitudinal axis, using a full 6-DOF model. The proposed control design methodology makes use of simple models of added mass and of dissipative hydrodynamic force acting on the vehicle. These models are both representative of the physics and sufficiently simple for control design and analysis. By considering an axisymmetric body, added-mass effects and dissipative hydrodynamic force are carefully accounted for via various adaptations, resulting in a modified apparent force independent of the vehicle's orientation and subsequently a nonlinear system with a triangular control structure. The proposed controller is also complemented with bounded integral correction actions to compensate for unavoidable model uncertainties and external disturbances. Compared to [1], here almost global asymptotical stability is achieved and the attitude is explicitly controlled. The proposed control approach endowed with a cascade inner-outer loop architecture can be seen as an 
extension of the thrust direction control paradigm that has been exploited for aerial vehicles [4], [6], [10], making a step towards a unified control approach for both aerial and underwater vehicles.

The paper is organized as follows. Notation and system modeling are provided in Section II. Then in the same section, a more simplified model for control design is derived for a class of axisymmetric underactuated underwater vehicles. Control design supported by rigourous stability analysis is presented in Section III. In section IV, comparative simulation results using a realistic model of a quasi-axisymmetric underwater vehicle illustrate the performance and robustness of the proposed control approach. Finally, a concluding section follows.

\section{NOTATION AND MODELING}

\section{A. Notation}

- $\left\{\mathbf{e}_{1}, \mathbf{e}_{2}, \mathbf{e}_{3}\right\}$ denotes the canonical basis of $\mathbb{R}^{3}$. $\mathbf{I}_{3}$ denotes the identity matrix of $\mathbb{R}^{3 \times 3}$. The notation $\mathbf{u}_{\times}, \forall \mathbf{u} \in \mathbb{R}^{3}$, denotes the skew-symmetric matrix associated with the cross product by $\mathbf{u}$, i.e., $\mathbf{u}_{\times} \mathbf{v}=\mathbf{u} \times \mathbf{v}, \forall \mathbf{v} \in \mathbb{R}^{3}$. The Euclidean norm in $\mathbb{R}^{n}$ and the transpose operator are, respectively, denoted as $|\cdot|$ and $(\cdot)^{\top} \cdot \pi_{\mathbf{x}}=\mathbf{I}_{3}-\mathbf{x} \mathbf{x}^{\top}$ is the projection onto the tangent space of the sphere $S^{2}$ of a point $\mathrm{x} \in S^{2}$. - Let $G$ and $B$ respectively denote the vehicle's center of mass $(\mathrm{CoM})$ and center of buoyancy $(\mathrm{CoB}), m$ its mass and $\mathbf{J}_{0}$ its inertia matrix. Let $g$ denote the gravity constant.

- Let $\{\mathcal{I}\}=\left\{O ; \vec{i}_{0}, \vec{j}_{0}, \vec{k}_{0}\right\}$ and $\{\mathcal{B}\}=\{B ; \vec{i}, \vec{j}, \vec{k}\}$ denote an inertial frame and a body-fixed frame attached to the vehicle, respectively.

- Let $\mathbf{r}_{G}$ denote the vector of coordinates expressed in $\{\mathcal{B}\}$ of the Euclidean vector $\overrightarrow{B G}$.

- The orientation of $\{\mathcal{B}\}$ relative to $\{\mathcal{I}\}$ is represented by the rotation matrix $\mathbf{R} \in \mathrm{SO}(3)$. Denote the position of the origin of the frame $\{\mathcal{B}\}$, expressed in $\{\mathcal{I}\}$ as $\xi \in \mathbb{R}^{3}$.

- The angular velocity of the frame $\{\mathcal{B}\}$ with respect to (w.r.t.) $\{\mathcal{I}\}$, expressed in $\{\mathcal{B}\}$, is denoted as $\Omega \in \mathbb{R}^{3}$.

- The vectors of coordinates of the linear velocity of the $\mathrm{CoB}$ $B$, expressed in the frames $\{\mathcal{I}\}$ and $\{\mathcal{B}\}$, are respectively denoted as $\mathbf{v} \in \mathbb{R}^{3}$ and $\mathbf{V} \in \mathbb{R}^{3}$.

- Let $\mathbf{v}_{f}$ and $\mathbf{V}_{f}$ denote the current velocity expressed in the frames $\{\mathcal{I}\}$ and $\{\mathcal{B}\}$, respectively. Denote $\mathbf{V}_{h} \triangleq \mathbf{V}-\mathbf{V}_{f}$ as the CoB's velocity w.r.t. the current.

\section{B. System modeling}

We consider a slender-body underactuated underwater vehicle with a body shape symmetric with respect to the longitudinal axis (e.g. the popular class of torpedo-shaped AUVs). The vehicle is endowed with four control inputs, namely a thrust force intensity $T \in \mathbb{R}$ of a control force vector $\mathbf{T}$ acting along the vehicle's longitudinal axis (i.e. $\left.\mathbf{T}=T \mathbf{e}_{1}\right)$ and three independent torque inputs $\boldsymbol{\Gamma} \in \mathbb{R}^{3}$ w.r.t. the $\mathrm{CoB}$ to control the vehicle's orientation. The thrust force is assumed to apply at a point lying on the $\{B ; \vec{i}\}$-axis so that it does not create any torque at the CoB.
The vehicle's rigid-body kinematic equations are

$$
\left\{\begin{array}{l}
\dot{\boldsymbol{\xi}}=\mathbf{R V} \\
\dot{\mathbf{R}}=\mathbf{R} \boldsymbol{\Omega}_{\times}
\end{array}\right.
$$

Defining the translational and rotational momentums as

$$
\left\{\begin{array}{l}
\mathbf{P}_{h} \triangleq \mathbf{M V}_{h}+\mathbf{D}^{\top} \boldsymbol{\Omega} \\
\mathbf{\Pi}_{h} \triangleq \mathbf{J} \boldsymbol{\Omega}+\mathbf{D} \mathbf{V}_{h}
\end{array}\right.
$$

with $\mathbf{M} \triangleq m \mathbf{I}_{3}+\mathbf{M}_{a}, \mathbf{J} \triangleq \mathbf{J}_{0}+\mathbf{J}_{a}, \mathbf{D} \triangleq m \mathbf{r}_{G \times}$, and $\mathbf{M}_{a}$, $\mathbf{J}_{a}$ respectively denoting the added-mass and added-inertia matrices. Then, according to the formulation of Leonard [8], the vehicle's dynamics are given by

$$
\left\{\begin{array}{l}
\dot{\mathbf{P}}_{h}=\mathbf{P}_{h} \times \boldsymbol{\Omega}+\mathbf{F}_{g b}+\mathbf{F}_{d}+T \mathbf{e}_{1} \\
\dot{\boldsymbol{\Pi}}_{h}=\boldsymbol{\Pi}_{h} \times \boldsymbol{\Omega}+\mathbf{P}_{h} \times \mathbf{V}_{h}+\boldsymbol{\Gamma}_{g}+\boldsymbol{\Gamma}_{d}+\boldsymbol{\Gamma}
\end{array}\right.
$$

where $\mathbf{F}_{g b} \triangleq \beta_{g b} \mathbf{R}^{\top} \mathbf{e}_{3}$, with $\beta_{g b} \triangleq m g-F_{b}$, is the sum of the gravity and buoyancy forces; $\boldsymbol{\Gamma}_{g} \triangleq m g \mathbf{r}_{G} \times \mathbf{R}^{\top} \mathbf{e}_{3}$ is the gravity torque; and $\mathbf{F}_{d}$ and $\boldsymbol{\Gamma}_{d}$ denote the dissipative hydrodynamic force and torque vectors expressed in the body-fixed frame $\{\mathcal{B}\}$.

The following classical model of $\mathbf{F}_{d}$ is used:

$$
\mathbf{F}_{d}\left(\mathbf{V}_{h}\right)=\underbrace{-\mathbf{K}_{d l} \mathbf{V}_{h}}_{\triangleq \mathbf{F}_{d l}\left(\mathbf{V}_{h}\right)} \underbrace{-\left|\mathbf{V}_{h}\right| \mathbf{K}_{d q} \mathbf{V}_{h}}_{\triangleq \mathbf{F}_{d q}\left(\mathbf{V}_{h}\right)}
$$

with the damping diagonal matrices

$$
\begin{aligned}
& \mathbf{K}_{d l}=\operatorname{diag}\left(k_{d l 1}, k_{d l 2}, k_{d l 2}\right) \\
& \mathbf{K}_{d q}=\operatorname{diag}\left(k_{d q 1}, k_{d q 2}, k_{d q 2}\right)
\end{aligned}
$$

The second and third diagonal components of $\mathbf{K}_{d l}$ and $\mathbf{K}_{d q}$ are equal due to the body symmetry about the longitudinal axis. Similarly, the added-mass matrix can also be modeled as a diagonal matrix with the same second and third diagonal components, i.e. $\mathbf{M}_{a}=\operatorname{diag}\left(m_{a 1}, m_{a 2}, m_{a 2}\right)$, with $m_{a 1} \ll$ $m_{a 2}$. Thus, the summed mass matrix $\mathbf{M}$ has the form $\mathbf{M}=$ $\operatorname{diag}\left(m_{1}, m_{2}, m_{2}\right)$ with $m_{1} \triangleq m+m_{a 1}, m_{2} \triangleq m+m_{a 2}$.

\section{Model for control design}

Due to the coupling matrix $\mathbf{D}$ involved in the definition (2) of the momentum terms and their dynamics (3), the translational and rotational dynamics are tightly coupled. These complex dynamic couplings are often neglected in the literature by neglecting all terms involving the matrix D using the fact that the distance between the CoB and $\mathrm{CoM}$ is small enough. This results in the following simpler control model that decouples the translational and rotational dynamics [1], [11], [12]:

$$
\begin{aligned}
\mathbf{M} \dot{\mathbf{V}} & =(\mathbf{M V}) \times \boldsymbol{\Omega}+\mathbf{F}_{g b}+\mathbf{F}_{d}(\mathbf{V})+T \mathbf{e}_{1}+\boldsymbol{\Delta}_{F} \\
\mathbf{J} \dot{\boldsymbol{\Omega}} & =(\mathbf{J} \boldsymbol{\Omega}) \times \boldsymbol{\Omega}+(\mathbf{M V}) \times \mathbf{V}+\boldsymbol{\Gamma}_{g}+\boldsymbol{\Gamma}_{d}+\boldsymbol{\Gamma}+\boldsymbol{\Delta}_{\Gamma}
\end{aligned}
$$

with the "disturbance" terms

$$
\begin{aligned}
& \boldsymbol{\Delta}_{F} \triangleq-(\mathbf{M V})_{\times} \boldsymbol{\Omega}-\mathbf{M} \boldsymbol{\Omega}_{\times} \mathbf{V}_{f}+\mathbf{F}_{d}\left(\mathbf{V}_{h}\right)-\mathbf{F}_{d}(\mathbf{V}) \\
& \boldsymbol{\Delta}_{\Gamma} \triangleq\left(\mathbf{M} \mathbf{V}_{h}\right) \times \mathbf{V}_{h}-(\mathbf{M V}) \times \mathbf{V}
\end{aligned}
$$

which are null if the current velocity is null. For the sake of simplicity, the disturbance term $\boldsymbol{\Delta}_{F}$ will not be considered for control design (i.e. $\boldsymbol{\Delta}_{F} \equiv \mathbf{0}$ ). However, integral corrections will be added so as to enhance control robustness w.r.t. unavoidable model errors and additive disturbances (i.e. current). 
In the sequel, the simplified dynamics (4) (with $\boldsymbol{\Delta}_{F} \equiv \mathbf{0}$ ) will be used for control design, whereas the dynamic equations (3) are still of use to simulate the vehicle's dynamics in the simulation section IV. Discrepancies between the two models represent an opportunity to test the robustness of the proposed controller.

Denote $\mathbf{P} \triangleq \mathbf{M V}$ and $\mathbf{p} \triangleq \mathbf{R P}=\mathbf{R M V}$ as the "simplified" translational momentums expressed in the bodyfixed frame and the inertial frame, respectively. One easily verifies from (4a) that the dynamics of $\mathbf{p}$ (i.e. $\dot{\mathbf{p}}$ ) does not depend on $\boldsymbol{\Omega}$.

According to the basic control methodology for thrustpropelled underactuated vehicles presented in [4], control solution may become implicit (or even ill-posed) if the external force expressed in the inertial frame $\mathbf{f}_{e} \triangleq \mathbf{R}\left(\mathbf{F}_{g b}+\right.$ $\left.\mathbf{F}_{d}(\mathbf{V})\right)=\beta_{g b} \mathbf{e}_{3}+\mathbf{R F}_{d}(\mathbf{V})$ depends strongly on the vehicle's orientation $\mathbf{R}$ but is not properly taken into account. The solution here proposed (similarly to the one developed in [10] for the control of axisymmetric aerial underactuated vehicles) consists in decomposing $\mathbf{F}_{d}(\mathbf{V})$ into two parts with the first one acting along $\mathbf{P}$ and the second one acting along $\mathbf{e}_{1}$. More precisely, one rewrites

$$
\begin{aligned}
& \mathbf{F}_{d l}(\mathbf{V})=-\frac{k_{d l 2}}{m_{2}} \mathbf{P}-\left(k_{d l 1}-\frac{m_{1} k_{d l 2}}{m_{2}}\right) V_{1} \mathbf{e}_{1} \\
& \mathbf{F}_{d q}(\mathbf{V})=-\frac{k_{d q 2}}{m_{2}}|\mathbf{V}| \mathbf{P}-\left(k_{d q 1}-\frac{m_{1} k_{d q 2}}{m_{2}}\right)|\mathbf{V}| V_{1} \mathbf{e}_{1}
\end{aligned}
$$

so that Eq. (4a) can be rewritten as

$$
\dot{\mathbf{P}}=\mathbf{P} \times \boldsymbol{\Omega}+\mathbf{F}_{g b}+\overline{\mathbf{F}}_{d l}+\overline{\mathbf{F}}_{d q}+\bar{T} \mathbf{e}_{1}
$$

with

$$
\left\{\begin{array}{l}
\overline{\mathbf{F}}_{d l} \triangleq-\beta_{d l} \mathbf{P}, \text { with } \beta_{d l} \triangleq \frac{k_{d l 2}}{m_{2}} \\
\overline{\mathbf{F}}_{d q} \triangleq-\beta_{d q}|\mathbf{V}| \mathbf{P}, \text { with } \beta_{d q} \triangleq \frac{k_{d q 2}}{m_{2}} \\
\bar{T} \triangleq T-\left(k_{d l 1}-\frac{m_{1} k_{d l 2}}{m_{2}}\right) V_{1}-\left(k_{d q 1}-\frac{m_{1} k_{d q 2}}{m_{2}}\right)|\mathbf{V}| V_{1}
\end{array}\right.
$$

Note that the quadratic drag force $\mathbf{R} \overline{\mathbf{F}}_{d q}$, expressed in the inertial frame $\{\mathcal{I}\}$, is not simply a function of $\mathbf{p}$ but also depends on the norm of $\mathbf{V}\left(=\mathbf{M}^{-1} \mathbf{R}^{\top} \mathbf{p}\right)$ and thus on the attitude $\mathbf{R}$. This in turn implies that the time derivative of $\mathbf{R} \overline{\mathbf{F}}_{d q}\left(=-\beta_{d q}\left|\mathbf{M}^{-1} \mathbf{R}^{\top} \mathbf{p}\right| \mathbf{p}\right)$ involves the angular velocity $\boldsymbol{\Omega}$, making the control design more delicate.

\section{TRAJECTORY TRACKING CONTROL DESIGN}

\section{A. Basic developments}

Let $\boldsymbol{\xi}_{r} \in \mathbb{R}^{3}$ denote a smooth differentiable reference position trajectory. Define $\mathbf{v}_{r} \triangleq \dot{\boldsymbol{\xi}}_{r} \in \mathbb{R}^{3}$ as the reference velocity expressed in the inertial frame $\{\mathcal{I}\}$. Assume that $\left|\mathbf{v}_{r}(t)\right| \geq c_{v}>0$ and also that $\mathbf{v}_{r}(t)$ and $\dot{\mathbf{v}}_{r}(t)$ remain bounded for all time $t>0$.

The position and velocity errors are defined as

$$
\left\{\begin{array}{l}
\tilde{\boldsymbol{\xi}} \triangleq \boldsymbol{\xi}-\boldsymbol{\xi}_{r} \\
\tilde{\mathbf{v}} \triangleq \mathbf{v}-\mathbf{v}_{r}
\end{array}\right.
$$

whereas the velocity error and the translational momentum error, both expressed in the body-fixed frame, are defined as

$$
\left\{\begin{array}{l}
\tilde{\mathbf{V}} \triangleq \mathbf{V}-\mathbf{R}^{\top} \mathbf{v}_{r}=\mathbf{R}^{\top} \tilde{\mathbf{v}} \\
\tilde{\mathbf{P}} \triangleq \mathbf{M} \tilde{\mathbf{V}}
\end{array}\right.
$$

Then, the control objective consists in stabilizing $(\tilde{\boldsymbol{\xi}}, \tilde{\mathbf{v}})$ or, equivalently, $(\tilde{\boldsymbol{\xi}}, \tilde{\mathbf{P}})$ about zero.

One easily verifies that

$$
\dot{\tilde{\xi}}=\mathbf{R} \tilde{\mathbf{V}}=\mathbf{R M}^{-1} \tilde{\mathbf{P}}
$$

On the other hand, using the decomposition

$$
\mathbf{M}=m_{2} \mathbf{I}_{3}+m_{12} \mathbf{e}_{1} \mathbf{e}_{1}^{\top}
$$

with $m_{12} \triangleq m_{1}-m_{2}=m_{a 1}-m_{a 2}<0$, one deduces from (5) and (7) that

$$
\begin{aligned}
\dot{\tilde{\mathbf{P}}}= & -\boldsymbol{\Omega}_{\times} \mathbf{P}+\mathbf{F}_{g b}+\overline{\mathbf{F}}_{d l}+\overline{\mathbf{F}}_{d q}+\bar{T} \mathbf{e}_{1} \\
& +\mathbf{M} \boldsymbol{\Omega}_{\times} \mathbf{R}^{\top} \mathbf{v}_{r}-\mathbf{M} \mathbf{R}^{\top} \dot{\mathbf{v}}_{r} \\
= & -\boldsymbol{\Omega}_{\times} \tilde{\mathbf{P}}-\boldsymbol{\Omega}_{\times} \mathbf{M} \mathbf{R}^{\top} \mathbf{v}_{r}+\mathbf{M} \boldsymbol{\Omega}_{\times} \mathbf{R}^{\top} \mathbf{v}_{r} \\
& +\mathbf{F}_{g b}+\overline{\mathbf{F}}_{d l}+\overline{\mathbf{F}}_{d q}-m_{2} \mathbf{R}^{\top} \dot{\mathbf{v}}_{r} \\
& +\left(\bar{T}-m_{12} \mathbf{e}_{1}^{\top} \mathbf{R}^{\top} \dot{\mathbf{v}}_{r}\right) \mathbf{e}_{1} \\
= & -\boldsymbol{\Omega}_{\times} \tilde{\mathbf{P}}-\beta_{d q}\left(|\mathbf{V}|-\left|\mathbf{v}_{r}\right|\right) \mathbf{P}+\alpha_{r} \mathbf{e}_{1 \times} \boldsymbol{\Omega} \\
& +\mathbf{R}^{\top}\left(\beta_{g b} \mathbf{e}_{3}-\beta_{d l} \mathbf{p}-\beta_{d q}\left|\mathbf{v}_{r}\right| \mathbf{p}-m_{2} \dot{\mathbf{v}}_{r}\right)+\left(\bar{T}-\dot{\alpha}_{r}\right) \mathbf{e}_{1}
\end{aligned}
$$

with

$$
\alpha_{r} \triangleq m_{12} \mathbf{e}_{1}^{\top} \mathbf{R}^{\top} \mathbf{v}_{r}
$$

The term $-\beta_{d q}\left(|\mathbf{V}|-\left|\mathbf{v}_{r}\right|\right) \mathbf{P}$ involved in the last equality of (9) requires further developments. Using the decomposition

$$
\tilde{\mathbf{P}}=\mathbf{P}-m_{2} \mathbf{R}^{\top} \mathbf{v}_{r}-\alpha_{r} \mathbf{e}_{1}
$$

one deduces

$$
\begin{aligned}
& \left(|\mathbf{V}|-\left|\mathbf{v}_{r}\right|\right) \mathbf{P} \\
& =-\left(|\mathbf{V}|-\left|\mathbf{v}_{r}\right|\right) \alpha_{r} \mathbf{e}_{1}+\left(|\mathbf{V}|-\left|\mathbf{v}_{r}\right|\right)\left(\tilde{\mathbf{P}}+m_{2} \mathbf{R}^{\top} \mathbf{v}_{r}\right) \\
& =-\left(|\mathbf{V}|-\left|\mathbf{v}_{r}\right|\right) \alpha_{r} \mathbf{e}_{1}+\frac{\left|\tilde{\mathbf{V}}+\mathbf{R}^{\top} \mathbf{v}_{r}\right|^{2}-\left|\mathbf{v}_{r}\right|^{2}}{|\mathbf{V}|+\left|\mathbf{v}_{r}\right|}\left(\tilde{\mathbf{P}}+m_{2} \mathbf{R}^{\top} \mathbf{v}_{r}\right) \\
& =-\left(|\mathbf{V}|-\left|\mathbf{v}_{r}\right|\right) \alpha_{r} \mathbf{e}_{1}+\frac{|\tilde{\mathbf{V}}|^{2}+2 \tilde{\mathbf{V}}^{\top} \mathbf{R}^{\top} \mathbf{v}_{r}}{|\mathbf{V}|+\left|\mathbf{v}_{r}\right|}\left(\tilde{\mathbf{P}}+m_{2} \mathbf{R}^{\top} \mathbf{v}_{r}\right)
\end{aligned}
$$

From (9) and (11) one obtains

$$
\begin{aligned}
\dot{\tilde{\mathbf{P}}}= & -\boldsymbol{\Omega}_{\times} \tilde{\mathbf{P}}-\beta_{d q} \frac{|\tilde{\mathbf{V}}|^{2}+2 \tilde{\mathbf{V}}^{\top} \mathbf{R}^{\top} \mathbf{v}_{r}}{|\mathbf{V}|+\left|\mathbf{v}_{r}\right|}\left(\tilde{\mathbf{P}}+m_{2} \mathbf{R}^{\top} \mathbf{v}_{r}\right) \\
& +\alpha_{r} \mathbf{e}_{1 \times} \boldsymbol{\Omega}+\mathbf{R}^{\top} \mathbf{f}_{p}+T_{p} \mathbf{e}_{1}
\end{aligned}
$$

with

$$
\left\{\begin{array}{l}
\mathbf{f}_{p} \triangleq \beta_{g b} \mathbf{e}_{3}-\beta_{d l} \mathbf{p}-\beta_{d q}\left|\mathbf{v}_{r}\right| \mathbf{p}-m_{2} \dot{\mathbf{v}}_{r} \\
T_{p} \triangleq \bar{T}-\dot{\alpha}_{r}+\beta_{d q}\left(|\mathbf{V}|-\left|\mathbf{v}_{r}\right|\right) \alpha_{r}
\end{array}\right.
$$

Let us call $\mathbf{f}_{p}$ the "apparent" force (expressed in the inertial frame). Note that its time derivative is independent of the angular velocity $\boldsymbol{\Omega}$ (i.e. $\dot{\mathbf{f}}_{p} \nsim \mathbf{\Omega}$ ), which is an important property for control design.

The proposed controller will be derived from Eqs. (8), (12) and (4b), which are in cascade form. In fact, the rotational dynamics given by (4b) are fully-actuated with a 3 -dimensional control torque vector $\boldsymbol{\Gamma}$ monitoring 3 degrees of freedom of rotation. It is thus straightforward to design an "inner-loop" controller that asymptotically stabilizes $\Omega$ about any bounded smooth desired angular velocity $\boldsymbol{\Omega}_{d}$, provided that the time derivative of the latter is available 
as feedforward. For instance, assuming that $\boldsymbol{\Delta}_{\Gamma}$ is constant, the following torque controller

$$
\begin{aligned}
\boldsymbol{\Gamma}= & -\mathbf{K}_{1} \tilde{\boldsymbol{\Omega}}-\mathbf{K}_{2} \mathbf{I}_{\tilde{\Omega}}+\mathbf{J} \dot{\boldsymbol{\Omega}}_{d} \\
& -(\mathbf{J} \boldsymbol{\Omega}) \times \boldsymbol{\Omega}_{d}-(\mathbf{M V}) \times \mathbf{V}-\boldsymbol{\Gamma}_{g}-\boldsymbol{\Gamma}_{d}
\end{aligned}
$$

with $\tilde{\boldsymbol{\Omega}} \triangleq \boldsymbol{\Omega}-\boldsymbol{\Omega}_{d}, \mathbf{I}_{\tilde{\Omega}}$ an integrator of $\tilde{\boldsymbol{\Omega}}$, and $\mathbf{K}_{1,2} \in \mathbb{R}^{3 \times 3}$ positive diagonal gain matrices, results in a closed-loop stable sub-system. Hence, the underlying idea for "outerloop" control design consists in using $\Omega$ as an intermediate control variable for the translational dynamics (i.e. Eq. (12)).

\section{B. Outer-loop control design}

Let $\mathbf{y} \triangleq \mathbf{K}_{\xi} \tilde{\boldsymbol{\xi}}$ denote the position error scaled by a diagonal positive gain matrix $\mathbf{K}_{\xi} \in \mathbb{R}^{3 \times 3}$. By introducing feedback terms (i.e. functions of $\tilde{\mathbf{P}}$ and $\tilde{\boldsymbol{\xi}}$ ), the translational error dynamics (12) can be rewritten as

$$
\begin{aligned}
\dot{\tilde{\mathbf{P}}}= & -\boldsymbol{\Omega}_{\times} \tilde{\mathbf{P}} \underbrace{-\mathbf{K}_{\mathbf{P}} \tilde{\mathbf{P}}-\mathbf{M}^{-1} \mathbf{R}^{\top} \mathbf{K}_{\xi}\left(h\left(|\mathbf{y}|^{2}\right) \mathbf{y}\right)}_{\text {feedback terms }} \\
& -\beta_{d q} \frac{|\tilde{\mathbf{V}}|^{2}+2 \tilde{\mathbf{V}}^{\top} \mathbf{R}^{\top} \mathbf{v}_{r}}{|\mathbf{V}|+\left|\mathbf{v}_{r}\right|}\left(\tilde{\mathbf{P}}+m_{2} \mathbf{R}^{\top} \mathbf{v}_{r}\right) \\
& +\alpha_{r} \mathbf{e}_{1 \times} \boldsymbol{\Omega}+\mathbf{R}^{\top} \overline{\mathbf{f}}_{p}+\bar{T}_{p} \mathbf{e}_{1}
\end{aligned}
$$

with $\mathbf{K}_{\mathrm{P}} \triangleq \operatorname{diag}\left(k_{p 1}+k_{p 2}, k_{p 1}, k_{p 1}\right), k_{p 1}, k_{p 2}>0, h(\cdot)$ denoting a smooth bounded strictly positive function defined on $[0,+\infty)$ such that for some positive numbers $\eta, \beta$

$$
\left\{\begin{array}{l}
\forall s \in \mathbb{R}, h\left(s^{2}\right) s<\eta \\
\forall s \in \mathbb{R}, \frac{\partial}{\partial s}\left(h\left(s^{2}\right) s\right)<\beta
\end{array}\right.
$$

and

$$
\left\{\begin{array}{l}
\overline{\mathbf{f}}_{p} \triangleq \mathbf{f}_{p}+k_{p 1}\left(\mathbf{p}-m_{2} \mathbf{v}_{r}\right)+\frac{1}{m_{2}} \mathbf{K}_{\xi}\left(h\left(|\mathbf{y}|^{2}\right) \mathbf{y}\right) \\
\bar{T}_{p} \triangleq T_{p}-k_{p 1} \alpha_{r}+k_{p 2} \tilde{P}_{1}-\frac{m_{12}}{m_{1} m_{2}} \mathbf{e}_{1}^{\top} \mathbf{R}^{\top} \mathbf{K}_{\xi}\left(h\left(|\mathbf{y}|^{2}\right) \mathbf{y}\right)
\end{array}\right.
$$

Note that the augmented apparent force $\overline{\mathbf{f}}_{p}$ does not depend on the vehicle's attitude $\mathbf{R}$ nor on $\boldsymbol{\Omega}$. Thus, its time derivative does not depend on the angular velocity $\Omega$.

If the outer-loop controller asymptotically stabilizes

$$
\varepsilon_{\mathrm{P}} \triangleq \alpha_{r} \mathbf{e}_{1 \times} \boldsymbol{\Omega}+\mathbf{R}^{\top} \overline{\mathbf{f}}_{p}+\bar{T}_{p} \mathbf{e}_{1}
$$

about zero, then the resulting zero dynamics of (15) are

$$
\begin{aligned}
\dot{\tilde{\mathbf{P}}}= & -\boldsymbol{\Omega}_{\times} \tilde{\mathbf{P}}-\mathbf{K}_{\mathrm{P}} \tilde{\mathbf{P}}-\mathbf{M}^{-1} \mathbf{R}^{\top} \mathbf{K}_{\xi}\left(h\left(|\mathbf{y}|^{2}\right) \mathbf{y}\right) \\
& -\beta_{d q} \frac{|\tilde{\mathbf{V}}|^{2}+2 \tilde{\mathbf{V}}^{\top} \mathbf{R}^{\top} \mathbf{v}_{r}}{|\mathbf{V}|+\left|\mathbf{v}_{r}\right|}\left(\tilde{\mathbf{P}}+m_{2} \mathbf{R}^{\top} \mathbf{v}_{r}\right)
\end{aligned}
$$

We show next that appropriate choices of gains $k_{p 1}$ and $k_{p 2}$ (i.e. $\mathbf{K}_{\mathrm{P}}$ ) will render the origin of the zero-dynamic timevarying system (8)+(18) globally asymptotically stable.

Lemma 1 Choose $k_{p 1}$ and $k_{p 2}$ such that

$$
\left\{\begin{array}{l}
k_{p 1}>\left(1+\frac{m_{2}}{2 m_{1}}\right)^{2} \beta_{d q} \sup \left(\left|\mathbf{v}_{r}\right|\right) \\
k_{p 2}>\left(1-\frac{m_{2}}{m_{1}}\right)^{2} \beta_{d q} \sup \left(\left|\mathbf{v}_{r}\right|\right)
\end{array}\right.
$$

then the equilibrium $(\tilde{\boldsymbol{\xi}}, \tilde{\mathbf{P}})=(\mathbf{0}, \mathbf{0})$ of the zero-dynamic system (8)+(18) is globally asymptotically stable.
Proof: The time derivative of the Lyapunov function candidate

$$
\mathcal{L} \triangleq \frac{1}{2}|\tilde{\mathbf{P}}|^{2}+\int_{0}^{|\mathbf{y}|} h\left(s^{2}\right) s \mathrm{~d} s
$$

satisfies

$$
\begin{aligned}
& \dot{\mathcal{L}}=-k_{p 1}|\tilde{\mathbf{P}}|^{2}-k_{p 2}\left|\tilde{P}_{1}\right|^{2} \\
& -\beta_{d q} \frac{|\tilde{\mathbf{V}}|^{2}+2 \tilde{\mathbf{V}}^{\top} \mathbf{R}^{\top} \mathbf{v}_{r}}{|\mathbf{V}|+\left|\mathbf{v}_{r}\right|}\left(|\tilde{\mathbf{P}}|^{2}+m_{2} \tilde{\mathbf{P}}^{\top} \mathbf{R}^{\top} \mathbf{v}_{r}\right) \\
& \leq-k_{p 1}|\tilde{\mathbf{P}}|^{2}-\frac{\beta_{d q}}{|\mathbf{V}|+\left|\mathbf{v}_{r}\right|}|\tilde{\mathbf{V}}|^{2}|\tilde{\mathbf{P}}|^{2} \\
& +\frac{2 \beta_{d q}\left|\mathbf{v}_{r}\right|}{|\mathbf{V}|+\left|\mathbf{v}_{r}\right|}|\tilde{\mathbf{V}}||\tilde{\mathbf{P}}|^{2}+\frac{\beta_{d q} m_{2}\left|\mathbf{v}_{r}\right|}{|\mathbf{V}|+\left|\mathbf{v}_{r}\right|}|\tilde{\mathbf{V}}|^{2}|\tilde{\mathbf{P}}| \\
& -k_{p 2}\left|\tilde{P}_{1}\right|^{2}-\frac{2 \beta_{d q} m_{2}^{2}}{|\mathbf{V}|+\left|\mathbf{v}_{r}\right|}\left(\tilde{\mathbf{V}}^{\top} \mathbf{R}^{\top} \mathbf{v}_{r}\right)^{2} \\
& +\frac{2 \beta_{d q} m_{2}\left|m_{12}\right|\left|\mathbf{v}_{r}\right|}{m_{1}\left(|\mathbf{V}|+\left|\mathbf{v}_{r}\right|\right)}\left|\tilde{P}_{1}\right|\left|\tilde{\mathbf{V}}^{\top} \mathbf{R}^{\top} \mathbf{v}_{r}\right|
\end{aligned}
$$

Using the relation $|\tilde{\mathbf{V}}| \leq \frac{1}{m_{1}}|\tilde{\mathbf{P}}|$ one then deduces

$$
\begin{aligned}
\dot{\mathcal{L}} \leq & -k_{p 1}|\tilde{\mathbf{P}}|^{2}-\frac{\beta_{d q}}{|\mathbf{V}|+\left|\mathbf{v}_{r}\right|}|\tilde{\mathbf{V}}|^{2}|\tilde{\mathbf{P}}|^{2}+\frac{\left(2+\frac{m_{2}}{m_{1}}\right) \beta_{d q}\left|\mathbf{v}_{r}\right|}{|\mathbf{V}|+\left|\mathbf{v}_{r}\right|}|\tilde{\mathbf{V}}||\tilde{\mathbf{P}}|^{2} \\
& -k_{p 2}\left|\tilde{P}_{1}\right|^{2}-\frac{2 \beta_{d q} m_{2}^{2}}{|\mathbf{V}|+\left|\mathbf{v}_{r}\right|}\left(\tilde{\mathbf{V}}^{\top} \mathbf{R}^{\top} \mathbf{v}_{r}\right)^{2} \\
& +\frac{2 \beta_{d q} m_{2}\left|m_{12}\right|\left|\mathbf{v}_{r}\right|}{m_{1}\left(|\mathbf{V}|+\left|\tilde{v}_{r}\right|\right)}\left|\tilde{P}_{1}\right|\left|\tilde{\mathbf{V}}^{\top} \mathbf{R}^{\top} \mathbf{v}_{r}\right|
\end{aligned}
$$

The gain condition (19) then ensures that all the negative quadratic terms dominate the cross terms, i.e.

$$
\begin{aligned}
k_{p 1}|\tilde{\mathbf{P}}|^{2}+\frac{\beta_{d q}}{|\mathbf{V}|+\left|\mathbf{v}_{r}\right|}|\tilde{\mathbf{V}}|^{2}|\tilde{\mathbf{P}}|^{2}>\frac{\left(2+\frac{m_{2}}{m_{1}}\right) \beta_{d q}\left|\mathbf{v}_{r}\right|}{|\mathbf{V}|+\left|\mathbf{v}_{r}\right|}|\tilde{\mathbf{V}}||\tilde{\mathbf{P}}|^{2} \\
k_{p 2}\left|\tilde{P}_{1}\right|^{2}+\frac{2 \beta_{d q} m_{2}^{2}}{|\mathbf{V}|+\left|\mathbf{v}_{r}\right|}\left(\tilde{\mathbf{V}}^{\top} \mathbf{R}^{\top} \mathbf{v}_{r}\right)^{2} \\
>\frac{2 \beta_{d q} m_{2}\left|m_{12}\right|\left|\mathbf{v}_{r}\right|}{m_{1}\left(|\mathbf{V}|+\left|\mathbf{v}_{r}\right|\right)}\left|\tilde{P}_{1}\right|\left|\tilde{\mathbf{V}}^{\top} \mathbf{R}^{\top} \mathbf{v}_{r}\right|
\end{aligned}
$$

This in turn implies the existence of a positive number $c$ such that $\dot{\mathcal{L}} \leq-c|\tilde{\mathbf{P}}|^{2}$. From there one can easily verify the boundedness of $\ddot{\mathcal{L}}$ (i.e. the uniform continuity of $\dot{\mathcal{L}}$ ) so that direct application of Barbalat's lemma then ensures the convergence of $\dot{\mathcal{L}}$ and, thus, of $\tilde{\mathbf{P}}$ to zero. From (18) and the convergence of $\tilde{\mathbf{P}}$ to zero, one deduces by application of the extended Barbalat's lemma [9] that $\dot{\tilde{\mathbf{P}}}$ also converges to zero, which in turn implies the convergence of $\mathbf{y}$ (i.e. $\tilde{\boldsymbol{\xi}}$ ) to zero. The remainder of the proof then follows.

Now the remaining task consists in designing the desired value $\Omega_{d}$ for $\Omega$ and the thrust intensity $\bar{T}_{p}$ (or equivalently T) to stabilize

$$
\alpha_{r} \mathbf{e}_{1 \times} \boldsymbol{\Omega}_{d}+\mathbf{R}^{\top} \overline{\mathbf{f}}_{p}+\bar{T}_{p} \mathbf{e}_{1} \rightarrow \mathbf{0}
$$

which is also equivalent to the stabilization of $\varepsilon_{\mathrm{P}}$ defined by (17) about zero as a consequence of the inner-loop controller that asymptotically stabilizes $\Omega$ about $\boldsymbol{\Omega}_{d}$.

Remark 1 By assumption $\mathbf{v}_{r}$ does not vanish for all time and $m_{12}=m_{a 1}-m_{a 2} \neq 0$ due to the slender-body form of the vehicle, one may expect that $\alpha_{r}$ defined by (10) does not vanish for all time either. Assuming that $\alpha_{r}(t) \neq 0 \forall t$, in view of (20) one may define the outer-loop controller as follows:

$$
\left\{\begin{array}{l}
\bar{T}_{p}=-\mathbf{e}_{1}^{\top} \mathbf{R}^{\top} \overline{\mathbf{f}}_{p} \\
\Omega_{d, 2}=-\frac{1}{\alpha_{r}} \mathbf{e}_{3}^{\top} \mathbf{R}^{\top} \overline{\mathbf{f}}_{p} \\
\Omega_{d, 3}=\frac{1}{\alpha_{r}} \mathbf{e}_{2}^{\top} \mathbf{R}^{\top} \overline{\mathbf{f}}_{p}
\end{array}\right.
$$


However, the outer-loop controller (21) leaves the attitude uncontrolled and ultimately guided by the system's zero dynamics, which may be excessively oscillating.

The outer-loop control solution proposed in this paper, by contrast, defines a desired direction $\mathbf{u} \in S^{2}$ for $\mathbf{R}^{\top} \boldsymbol{\gamma}$ with $\gamma \triangleq \overline{\mathbf{f}}_{p} /\left|\overline{\mathbf{f}}_{p}\right| \in S^{2}$ representing the direction of $\overline{\mathbf{f}}_{p}$, where $\mathbf{u}$ is obtained by integration of the following differential equation

$$
\dot{\mathbf{u}}=\mathbf{u} \times \boldsymbol{\Omega}_{u}, \quad \mathbf{u}(0)=-\mathbf{e}_{1}
$$

with $\boldsymbol{\Omega}_{u}$ an augmented control input to be designed thereafter. For instance, assuming that $\overline{\mathbf{f}}_{p}$ does not vanish for all time, one then verifies that

$$
\dot{\gamma}=\gamma \times \Omega_{\gamma}
$$

with $\boldsymbol{\Omega}_{\gamma} \triangleq-\frac{\overline{\mathbf{f}}_{p} \times \dot{\overline{\mathbf{f}}}_{p}}{\left|\overline{\mathbf{f}}_{p}\right|^{2}}$, which does not depend on $\boldsymbol{\Omega}$. In view of the expression of $\overline{\mathbf{f}}_{p}$ in (16), one ensures that $\boldsymbol{\Omega}_{\gamma}$ does not depend on the angular velocity $\Omega$.

Lemma 2 Assume that initially $\left(\mathbf{R}^{\top} \boldsymbol{\gamma}\right)(0) \neq-\mathbf{u}(0)$ and that the inner-loop controller ensures the convergence of $\tilde{\boldsymbol{\Omega}}$ about zero and also its boundedness. Then, by setting the following constraint

$$
\pi_{\mathbf{u}}\left(\boldsymbol{\Omega}_{d}-\boldsymbol{\Omega}_{u}\right)=-\pi_{\mathbf{u}}\left(\mathbf{R}^{\top} \boldsymbol{\Omega}_{\gamma}\right)-\frac{k_{u}\left(\mathbf{R}^{\top} \boldsymbol{\gamma} \times \mathbf{u}\right)}{1+\mathbf{u}^{\top} \mathbf{R}^{\top} \boldsymbol{\gamma}}
$$

with $k_{u}>0$, one ensures that $\mathbf{R}^{\top} \gamma$ converges asymptotically to $\mathbf{u}$.

Proof: Using (22), (23) and (24), the time derivative of the positive function $\mathcal{L}_{1} \triangleq 1-\mathbf{u}^{\top} \mathbf{R}^{\top} \boldsymbol{\gamma}$ verifies

$$
\begin{aligned}
\dot{\mathcal{L}}_{1} & =\left(\boldsymbol{\Omega}-\boldsymbol{\Omega}_{u}+\mathbf{R}^{\top} \boldsymbol{\Omega}_{\gamma}\right)^{\top}\left(\mathbf{R}^{\top} \boldsymbol{\gamma} \times \mathbf{u}\right) \\
& =\tilde{\boldsymbol{\Omega}}^{\top}\left(\mathbf{R}^{\top} \boldsymbol{\gamma} \times \mathbf{u}\right)+\left(\boldsymbol{\Omega}_{d}-\boldsymbol{\Omega}_{u}+\mathbf{R}^{\top} \boldsymbol{\Omega}_{\gamma}\right)^{\top}\left(\mathbf{R}^{\top} \boldsymbol{\gamma} \times \mathbf{u}\right) \\
& =\tilde{\boldsymbol{\Omega}}^{\top}\left(\mathbf{R}^{\top} \boldsymbol{\gamma} \times \mathbf{u}\right)-k_{u} \frac{\left|\mathbf{R}^{\top} \boldsymbol{\gamma} \times \mathbf{u}\right|^{2}}{1+\mathbf{u}^{\top} \mathbf{R}^{\top} \boldsymbol{\gamma}}
\end{aligned}
$$

where the first term $\tilde{\boldsymbol{\Omega}}^{\top}\left(\mathbf{R}^{\top} \boldsymbol{\gamma} \times \mathbf{u}\right)$ is bounded thanks to the boundedness of $\tilde{\boldsymbol{\Omega}}$, whereas the division by $1+\mathbf{u}^{\top} \mathbf{R}^{\top} \boldsymbol{\gamma}$ in the second term prevents $\mathbf{R}^{\top} \boldsymbol{\gamma}$ from tending close to $-\mathbf{u}$ since $\dot{\mathcal{L}}_{1}$ tends to $-\infty$ in this case.

Using the relation $\left|\mathbf{R}^{\top} \boldsymbol{\gamma} \times \mathbf{u}\right|^{2}+\left(\mathbf{u}^{\top} \mathbf{R}^{\top} \boldsymbol{\gamma}\right)^{2}=1$, one then deduces that

$$
\dot{\mathcal{L}}_{1}=\tilde{\boldsymbol{\Omega}}^{\top}\left(\mathbf{R}^{\top} \boldsymbol{\gamma} \times \mathbf{u}\right)-k_{u} \mathcal{L}_{1}
$$

Since $\tilde{\boldsymbol{\Omega}}$ converges to zero, the application of the singular perturbation theory then ensures the convergence of $\mathcal{L}_{1}$ to zero or equivalently of $\mathbf{R}^{\top} \boldsymbol{\gamma}$ to $\mathbf{u}$.

Now the main result of this section can be stated.

Proposition 1 Let $\bar{T}_{p}$ and $\overline{\mathbf{f}}_{p}$ be defined by (16)+(13) and $\alpha_{r}$ be defined by (10). Apply the outer-loop controller

$$
\left\{\begin{array}{l}
\bar{T}_{p}=-\mathbf{e}_{1}^{\top} \mathbf{R}^{\top} \overline{\mathbf{f}}_{p} \\
\boldsymbol{\Omega}_{d}=\frac{\left|\overline{\mathbf{f}}_{p}\right|}{\alpha_{r}} \mathbf{e}_{1} \times \mathbf{u}-\frac{k_{u}\left(\mathbf{R}^{\top} \boldsymbol{\gamma} \times \mathbf{u}\right)}{1+\mathbf{u}^{\top} \mathbf{R}^{\top} \boldsymbol{\gamma}}+\lambda \mathbf{e}_{1}
\end{array}\right.
$$

where $\mathbf{u}$ is obtained by integration of (22) with the augmented control input $\boldsymbol{\Omega}_{u}$ given by

$$
\boldsymbol{\Omega}_{u}=\frac{\left|\overline{\mathbf{f}}_{p}\right|}{\alpha_{r}} \mathbf{e}_{1} \times \mathbf{u}+\pi_{\mathbf{u}}\left(\mathbf{R}^{\top} \boldsymbol{\Omega}_{\gamma}\right)+\lambda \pi_{\mathbf{u}} \mathbf{e}_{1}
$$

and $\lambda(\cdot)$ is a function that can be independently assigned for other control objective related to the roll motion. Assume that $\alpha_{r} \neq 0$ and $\left|\overline{\mathbf{f}}_{p}\right|>0$ for all time. Assume that all conditions and assumptions in Lemmas 1 and 2 hold. Then, the equilibrium $\left(\boldsymbol{\xi}, \mathbf{v}, \mathbf{R}^{\top} \boldsymbol{\gamma}\right)=\left(\boldsymbol{\xi}_{r}, \mathbf{v}_{r}, \mathbf{u}\right)$ is almost globally asymptotically stable.

The proof of this proposition is a direct result of Lemmas 1 and 2.

Remark 2 In the case $m_{12}<0, \alpha_{r}$ is nominally negative. So if $\boldsymbol{\Omega}_{\gamma}$ is not too large, the term $\frac{\left|\overrightarrow{\mathbf{p}}_{\alpha}\right|}{\alpha_{r}} \mathbf{e}_{1} \times \mathbf{u}$ involved in (26) allows $\mathbf{u}$ not to depart too far from $-\mathbf{e}_{1}$. This can be justified by examining the derivative of $1+\mathbf{e}_{1}^{\top} \mathbf{u}$ :

$$
\frac{\mathrm{d}}{\mathrm{dt}}\left(1+\mathbf{e}_{1}^{\top} \mathbf{u}\right)=\underbrace{\frac{\left|\overline{\mathbf{f}}_{p}\right|}{\alpha_{r}}\left|\mathbf{e}_{1} \times \mathbf{u}\right|^{2}}_{\leq 0}+\left(\mathbf{e}_{1} \times \mathbf{u}\right)^{\top}\left(\mathbf{R}^{\top} \boldsymbol{\Omega}_{\gamma}\right)
$$

Remark 3 Since $\alpha_{r}$ may evolve near zero during a transient phase, to avoid the division by zero we suggest to replace the expressions of $\boldsymbol{\Omega}_{d}$ and $\boldsymbol{\Omega}_{u}$ given in (25) and (26) by

$$
\left\{\begin{array}{l}
\boldsymbol{\Omega}_{d}=\frac{\left|\overline{\mathbf{f}}_{p}\right|}{\sigma\left(\alpha_{r}\right)} \mathbf{e}_{1} \times \mathbf{u}-\frac{k_{u}\left(\mathbf{R}^{\top} \boldsymbol{\gamma} \times \mathbf{u}\right)}{1+\mathbf{u}^{\top} \mathbf{R}^{\top} \boldsymbol{\gamma}}+\lambda \mathbf{e}_{1} \\
\boldsymbol{\Omega}_{u}=\frac{\left|\overline{\mathbf{f}}_{p}\right|}{\sigma\left(\alpha_{r}\right)} \mathbf{e}_{1} \times \mathbf{u}+\pi_{\mathbf{u}}\left(\mathbf{R}^{\top} \boldsymbol{\Omega}_{\gamma}\right)+\lambda \pi_{\mathbf{u}} \mathbf{e}_{1}
\end{array}\right.
$$

with $\sigma(\cdot)$ given by

$$
\sigma(x)=\left\{\begin{array}{lll}
x & \text { if } & |x|>\varepsilon \\
\varepsilon & \text { if } & 0<x \leq \varepsilon \\
-\varepsilon & \text { if } & 0 \geq x \geq-\varepsilon
\end{array}\right.
$$

with $\varepsilon$ a small positive number. With this modification, relation (24) is always satisfied and, thus, the convergence of $\mathbf{R}^{\top} \gamma$ about $\mathbf{u}$ is still ensured.

\section{Outer-loop control design with integral term}

In practice it is often desirable to complement the control action with a position error integral correction term in order to compensate for model uncertainties and external disturbances. Bearing in mind that classical integrator of $\tilde{\xi}$ is often prone to the well-known phenomenon of integrator windup that may cause large overshoots of the position tracking error, we make use of the following bounded nonlinear secondorder integrator of $\tilde{\boldsymbol{\xi}}$ (initially proposed in [5]):

$$
\ddot{\mathbf{I}}_{\tilde{\xi}}=-2 k_{\mathrm{vI}} \dot{\mathbf{I}}_{\tilde{\xi}}+\operatorname{sat}_{\ddot{I}_{\text {max }} / 2}\left(k_{\mathrm{pI}}\left(-\mathbf{I}_{\tilde{\xi}}+\operatorname{sat}_{\delta_{\mathrm{I}}}\left(\mathbf{I}_{\tilde{\xi}}+\tilde{\boldsymbol{\xi}}\right)\right)\right)
$$

with $\ddot{\mathrm{I}}_{\text {max }}, \delta_{\mathrm{I}}, k_{\mathrm{pI}}, k_{\mathrm{vI}}$ denoting positive constants and with initial conditions satisfying $\left|\mathbf{I}_{\tilde{\xi}}(0)\right|<\delta_{\mathrm{I}}+\ddot{\mathrm{I}}_{\max } /\left(2 k_{\mathrm{vI}}^{2}\right)$ and $\left|\dot{\mathbf{I}}_{\tilde{\xi}}(0)\right|<\ddot{\mathrm{I}}_{\max } /\left(2 k_{\mathrm{vI}}\right)$. As specified in [5], the ultimate upper-bounds of $\left|\mathbf{I}_{\tilde{\xi}}\right|,\left|\dot{\mathbf{I}}_{\tilde{\xi}}\right|$, and $\left|\ddot{\mathbf{I}}_{\tilde{\xi}}\right|$ are $\delta_{\mathrm{I}}+\ddot{\mathrm{I}}_{\max } /\left(2 k_{\mathrm{vI}}^{2}\right)$, $\ddot{\mathrm{I}}_{\max } /\left(2 k_{\mathrm{vI}}\right)$, and $\ddot{\mathrm{I}}_{\max }$, respectively.

Define the augmented reference variables

$$
\left\{\begin{array}{l}
\overline{\boldsymbol{\xi}}_{r} \triangleq \boldsymbol{\xi}_{r}-\mathbf{K}_{\mathrm{I}} \mathbf{I}_{\tilde{\xi}} \\
\overline{\mathbf{v}}_{r} \triangleq \mathbf{v}_{r}-\mathbf{K}_{\mathrm{I}} \dot{\mathbf{I}}_{\tilde{\xi}}
\end{array}\right.
$$


with $\mathbf{K}_{\mathrm{I}} \in \mathbb{R}^{3 \times 3}$ a diagonal positive integral gain matrix. Define also the augmented error terms (compared to (6)-(7))

$$
\begin{cases}\overline{\tilde{\boldsymbol{\xi}}} \triangleq \boldsymbol{\xi}-\overline{\boldsymbol{\xi}}_{r} & =\tilde{\boldsymbol{\xi}}+\mathbf{K}_{\mathrm{I}} \mathbf{I}_{\tilde{\xi}} \\ \overline{\tilde{\mathbf{v}}} \triangleq \mathbf{v}-\overline{\mathbf{v}}_{r} & =\tilde{\mathbf{v}}+\mathbf{K}_{\mathrm{I}} \dot{\mathbf{I}}_{\tilde{\xi}} \\ \overline{\tilde{\mathbf{V}}} \triangleq \mathbf{V}-\mathbf{R}^{\top} \overline{\mathbf{v}}_{r} & =\mathbf{R}^{\top} \overline{\tilde{\mathbf{v}}} \\ \overline{\tilde{\mathbf{P}}} \triangleq \mathbf{M} \overline{\tilde{\mathbf{V}}} & =\mathbf{P}-\mathbf{M} \mathbf{R}^{\top} \overline{\mathbf{v}}_{r}\end{cases}
$$

One verifies that

$$
\dot{\overline{\tilde{\xi}}}=\mathbf{R} \mathbf{M}^{-1} \overline{\tilde{\mathbf{P}}}
$$

On the other hand, analogously to the developments carried out in (9)-(13) one deduces

$$
\begin{aligned}
\dot{\overline{\mathbf{P}}}= & -\boldsymbol{\Omega}_{\times} \overline{\tilde{\mathbf{P}}} \\
& -\beta_{d q} \frac{|\overline{\tilde{\mathbf{V}}}|^{2}+2 \overline{\tilde{\mathbf{V}}}^{\top} \mathbf{R}^{\top} \overline{\mathbf{v}}_{r}}{|\mathbf{V}|+\left|\overline{\mathbf{v}}_{r}\right|}\left(\overline{\tilde{\mathbf{P}}}+m_{2} \mathbf{R}^{\top} \overline{\mathbf{v}}_{r}\right) \\
& +\alpha_{r} \mathbf{e}_{1 \times} \boldsymbol{\Omega}+\mathbf{R}^{\top} \mathbf{f}_{p}+T_{p} \mathbf{e}_{1}
\end{aligned}
$$

where $\alpha_{r}$ is now defined by (instead of (10))

$$
\alpha_{r} \triangleq m_{12} \mathbf{e}_{1}^{\top} \mathbf{R}^{\top} \overline{\mathbf{v}}_{r}
$$

and $\mathbf{f}_{p}$ and $T_{p}$ are defined by (instead of (13))

$$
\left\{\begin{array}{l}
\mathbf{f}_{p} \triangleq \beta_{g b} \mathbf{e}_{3}-\beta_{d l} \mathbf{p}-\beta_{d q}\left|\overline{\mathbf{v}}_{r}\right| \mathbf{p}-m_{2} \dot{\overline{\mathbf{v}}}_{r} \\
T_{p} \triangleq \bar{T}-\dot{\alpha}_{r}+\beta_{d q}\left(|\mathbf{V}|-\left|\overline{\mathbf{v}}_{r}\right|\right) \alpha_{r}
\end{array}\right.
$$

Then, similarly to Eq. (15), Eq. (33) can be rewritten as

$$
\begin{aligned}
\dot{\overline{\tilde{\mathbf{P}}}}= & -\boldsymbol{\Omega}_{\times} \overline{\tilde{\mathbf{P}}}-\mathbf{K}_{\mathrm{P}} \overline{\tilde{\mathbf{P}}}-\mathbf{M}^{-1} \mathbf{R}^{\top} \mathbf{K}_{\xi}\left(h\left(|\overline{\mathbf{y}}|^{2}\right) \overline{\mathbf{y}}\right) \\
& \left.-\beta_{d q} \frac{|\overline{\tilde{\mathbf{V}}}|^{2}+2 \overline{\tilde{\mathbf{V}}}^{\top} \mathbf{R}^{\top} \overline{\mathbf{v}}_{r}}{|\mathbf{V}|+\left|\overline{\tilde{\mathbf{P}}}+\overline{\mathbf{v}}_{r}\right|}{m_{2}}^{\top} \overline{\mathbf{R}}_{r}\right) \\
& +\alpha_{r} \mathbf{e}_{1 \times} \boldsymbol{\Omega}+\mathbf{R}^{\top} \overline{\mathbf{f}}_{p}+\bar{T}_{p} \mathbf{e}_{1}
\end{aligned}
$$

with $\overline{\mathbf{y}} \triangleq \mathbf{K}_{\xi} \overline{\tilde{\boldsymbol{\xi}}}, \mathbf{K}_{\xi}$ a diagonal positive gain matrix, $\mathbf{K}_{\mathrm{P}} \triangleq$ $\operatorname{diag}\left(k_{p 1}+k_{p 2}, k_{p 1}, k_{p 1}\right)$ (with $k_{p 1}, k_{p 2}>0$ ), and $\overline{\mathbf{f}}_{p}$ and $\bar{T}_{p}$ defined by (instead of (16))

$$
\left\{\begin{array}{l}
\overline{\mathbf{f}}_{p} \triangleq \mathbf{f}_{p}+k_{p 1}\left(\mathbf{p}-m_{2} \overline{\mathbf{v}}_{r}\right)+\frac{1}{m_{2}} \mathbf{K}_{\xi}\left(h\left(|\overline{\mathbf{y}}|^{2}\right) \overline{\mathbf{y}}\right) \\
\bar{T}_{p} \triangleq T_{p}-k_{p 1} \alpha_{r}+k_{p 2} \overline{\tilde{P}}_{1}-\frac{m_{12}}{m_{1} m_{2}} \mathbf{e}_{1}^{\top} \mathbf{R}^{\top} \mathbf{K}_{\xi}\left(h\left(|\overline{\mathbf{y}}|^{2}\right) \overline{\mathbf{y}}\right)
\end{array}\right.
$$

Interestingly, Eqs. (32) and (36) have identical form as Eqs. (8) and (15), respectively. Therefore, similar outer-loop control expressions together with associated stability result as in Proposition 1 can be straightforwardly stated.

Proposition 2 Let $\bar{T}_{p}$ and $\overline{\mathbf{f}}_{p}$ be defined by (37)+(35) and $\alpha_{r}$ be defined by (34). Apply the outer-loop controller (25) where the involved term $\mathbf{u}$ is obtained by integration of (22) with the augmented control input $\Omega_{u}$ given by (26). Choose $k_{p 1}$ and $k_{p 2}$ such that

$$
\left\{\begin{array}{l}
k_{p 1}>\left(1+\frac{m_{2}}{2 m_{1}}\right)^{2} \beta_{d q}\left(\sup \left(\left|\mathbf{v}_{r}\right|\right)+\frac{\ddot{I}_{\text {max }}}{2 k_{\mathrm{vI}}}\right) \\
k_{p 2}>\left(1-\frac{m_{2}}{m_{1}}\right)^{2} \beta_{d q}\left(\sup \left(\left|\mathbf{v}_{r}\right|\right)+\frac{\ddot{\mathrm{I}}_{\text {max }}}{2 k_{\mathrm{vI}}}\right)
\end{array}\right.
$$

Assume that $\alpha_{r} \neq 0$ and $\left|\overline{\mathbf{f}}_{p}\right|>0$ for all time. Assume that all conditions and assumptions in Lemma 2 hold. Then, the equilibrium $\left(\mathbf{I}_{\tilde{\xi}}, \dot{\mathbf{I}}_{\tilde{\xi}}, \boldsymbol{\xi}, \mathbf{v}, \mathbf{R}^{\top} \boldsymbol{\gamma}\right)=\left(\mathbf{0}, \mathbf{0}, \boldsymbol{\xi}_{r}, \mathbf{v}_{r}, \mathbf{u}\right)$ is almost globally asymptotically stable.

Proof: The proof of this proposition is almost identical to that of Proposition 1. Indeed, Lemma 2 and the outerloop control expressions (25)-(26) ensure the convergence of the term $\alpha_{r} \mathbf{e}_{1 \times} \boldsymbol{\Omega}+\mathbf{R}^{\top} \overline{\mathbf{f}}_{p}+\bar{T}_{p} \mathbf{e}_{1}$ involved in (36) to zero. Then, similarly to the proof of Lemma 1, using (38) and the fact that $\sup \left(\left|\overline{\mathbf{v}}_{r}\right|\right) \leq \sup \left(\left|\mathbf{v}_{r}\right|\right)+\sup \left(\mathbf{I}_{\tilde{\xi}}\right) \leq$ $\sup \left(\left|\mathbf{v}_{r}\right|\right)+\ddot{\mathrm{I}}_{\max } /\left(2 k_{\mathrm{vI}}\right)$ one easily deduces that the equilibrium $(\tilde{\tilde{\boldsymbol{\xi}}}, \overline{\tilde{\mathbf{P}}})=(\mathbf{0}, \mathbf{0})$ of the zero dynamics of $(32)+(36)$ is globally asymptotically stable. Then, from the definition (31) of $\overline{\tilde{\boldsymbol{\xi}}}$ and $\overline{\tilde{\mathbf{v}}}$ the proof of convergence of $\left(\mathbf{I}_{\tilde{\xi}}, \dot{\mathbf{I}}_{\tilde{\xi}}, \tilde{\boldsymbol{\xi}}, \tilde{\mathbf{v}}\right)$ to zero follows the same lines as the proof of Lemma 3.2 in

\begin{tabular}{|c|c|c|c|}
\hline Specification & \multicolumn{3}{|c|}{ Numerical value } \\
\hline Mass $m[k g]$ & \multicolumn{3}{|c|}{100} \\
\hline$F_{b}[N]$ & \multicolumn{3}{|c|}{$m g$} \\
\hline $\mathbf{r}_{G}[m]$ & \multicolumn{3}{|c|}{$\left.\begin{array}{ll}0 & 0.01\end{array}\right]^{\top}$} \\
\hline & {$[10$} & 1 & 27 \\
\hline \multirow[t]{3}{*}{$\mathbf{J}_{0}\left[k g \cdot m^{2}\right]$} & 1 & 100 & 1 \\
\hline & {$[2$} & 1 & 90 \\
\hline & $\lceil 32.6$ & 3 & 4 \\
\hline \multirow[t]{3}{*}{$\mathbf{M}_{a}[k g]$} & 3 & 484.8 & 1 \\
\hline & 4 & 1 & 394 \\
\hline & 12.7 & 5 & 10 \\
\hline \multirow[t]{2}{*}{$\mathbf{J}_{a}\left[k g \cdot m^{2}\right]$} & 5 & 133.4 & 8 \\
\hline & 10 & 8 & 113.1 \\
\hline $\mathbf{K}_{d l}\left[k g . s^{-1}\right]$ & diag & $1.5,7.5$ & $5.5)$ \\
\hline $\mathbf{K}_{d q}\left[\mathrm{~kg} \cdot \mathrm{m}^{-1}\right]$ & $\operatorname{diag}(3$ & , 341.8, & $285.5)$ \\
\hline $\mathbf{K}_{\Omega l}\left[k g \cdot m^{2} \cdot s^{-1}\right]$ & & $\mathrm{g}(1,35$, & 10) \\
\hline $\mathbf{K}_{\Omega q}[N . m]$ & diag & 10,352 , & 437) \\
\hline
\end{tabular}
[5]. The remainder of the proof then directly follows.

\section{Simulation RESUlts}

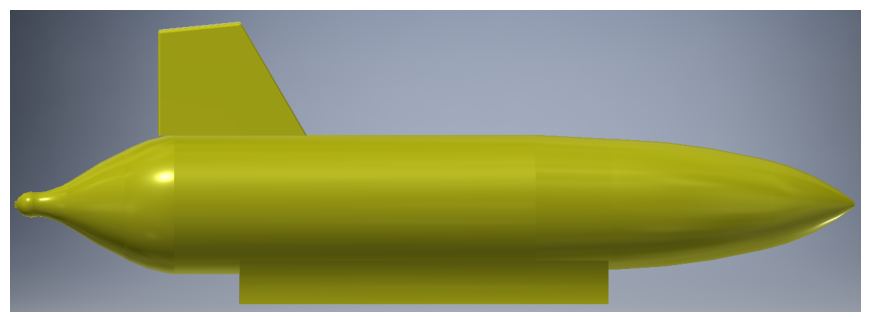

Fig. 1. Quasi-axisymmetric shape of the simulated vehicle with the main body's dimension $2.7[\mathrm{~m}] \times 0.45[\mathrm{~m}]$

In this section the performance and robustness of the proposed control approach are validated through simulation conducted on a realistic model of a quasi-axisymmetric underactuated vehicle. The vehicle's body shape, as depicted in Fig. 1, is not perfectly symmetric along the longitudinal axis due to a large rudder and a lower base.

TABLE I

SPECIFICATIONS OF THE SIMULATED VEHICLE

The simulated dynamics are given by (3). Physical parameters of the simulated vehicle are provided in Tab. I, where the added-mass, added-inertia and damping coefficients are roughly identified from the given shape. Note that the second and third components of the added-mass matrix $\mathbf{M}_{a}$ and 
of the damping matrix $\mathbf{K}_{d q}$ are significantly different. This represents an opportunity to test the robustness of proposed controller w.r.t. such a violation of the axisymmetric assumption used for control design. For control implementation, an approximate axisymmetric model is used with the following estimated and approximated parameters:

$$
\left\{\begin{array}{cl}
\hat{\mathbf{M}}_{a} & =\operatorname{diag}(30,440,440)[\mathrm{kg}] \\
\hat{\mathbf{J}}=\hat{\mathbf{J}}_{0}+\hat{\mathbf{J}}_{a} & =\operatorname{diag}(20,200,190)\left[\mathrm{kg} \cdot \mathrm{m}^{2}\right] \\
\hat{\mathbf{K}}_{d l} & =\operatorname{diag}(1,6,6)\left[\mathrm{kg} \cdot \mathrm{s}^{-1}\right] \\
\hat{\mathbf{K}}_{d q} & =\operatorname{diag}(30,310,310)\left[\mathrm{kg} \cdot \mathrm{m}^{-1}\right]
\end{array}\right.
$$

One can notice that these estimated terms are quite different from the corresponding "real" ones given in Tab. I. In addition, a non-null current velocity $\mathbf{v}_{f}=$ $\left[\begin{array}{lll}-0.1 & 0.2 & 0.05\end{array}\right]^{\top}\left[\mathrm{m.s}^{-2}\right]$ is introduced, allowing us to test the control robustness w.r.t. both model uncertainties and external disturbances.

The simulated model of the dissipative torque $\boldsymbol{\Gamma}_{d}$ is given by $\boldsymbol{\Gamma}_{d}=-\mathbf{K}_{\Omega l} \boldsymbol{\Omega}-\mathbf{K}_{\Omega q}|\boldsymbol{\Omega}| \boldsymbol{\Omega}$, with the damping matrices $\mathbf{K}_{\Omega l}$ and $\mathbf{K}_{\Omega q}$ specified in Tab. I, whereas in the inner-loop torque control expression (14) the estimate of this dissipative torque is simply set equal to zero (i.e. $\hat{\boldsymbol{\Gamma}}_{d}=\mathbf{0}$ ) so that no prior knowledge of this torque is required for control implementation.

The gains and parameters involved in the proposed controller are chosen as follows:

$$
\left\{\begin{array}{l}
k_{p 1}=5.6154, k_{p 2}=8, \\
\mathbf{K}_{\xi}=\operatorname{diag}(900,1870,1870), \mathbf{K}_{\mathrm{I}}=0.5 \mathbf{I}_{3}, \\
h(s)=\frac{1}{\sqrt{1+s / \eta^{2}}}, \eta=\hat{m}_{2}=540 \\
k_{u}=2, \\
\sigma(\cdot) \text { given by }(28) \text { with } \varepsilon=0.1 \\
\lambda=\mathbf{e}_{1}^{\top}\left(\mathbf{e}_{3} \times \mathbf{R}^{\top} \mathbf{e}_{3}\right),
\end{array}\right.
$$

Note that the above expression of $\lambda$ allows for maintaining roll angle near zero. Limitations of the actuators are also taken into account by saturating the applied thrust force and torque control inputs according to the following inequality constraints $|T| \leq 200[N]$ and $\left|\Gamma_{i=1,2,3}\right| \leq 160[N . m]$.

The reference trajectory is a horizontal circular trajectory with radius $20[\mathrm{~m}]$ and constant tangential speed $1[\mathrm{~m} / \mathrm{s}]$ (i.e $\left.\left|\mathbf{v}_{r}\right|=1[\mathrm{~m} / \mathrm{s}]\right)$. More precisely,

$$
\boldsymbol{\xi}_{r}(t)=\left[\begin{array}{lll}
20 \sin \left(\frac{t}{20}\right) & 20\left(\cos \left(\frac{t}{20}\right)-1\right) & 0
\end{array}\right]^{\top}[m]
$$

Note that $\boldsymbol{\xi}_{r}(0)=\mathbf{0}$ and $\mathbf{v}_{r}(0)=\dot{\boldsymbol{\xi}}_{r}(0)=\mathbf{e}_{1}$. The initial conditions are chosen such that initial errors are relatively large, namely

$$
\left\{\begin{array}{l}
\boldsymbol{\xi}(0)=\left[\begin{array}{lll}
-20 & 15 & 10
\end{array}\right]^{\top}, \mathbf{v}(0)=\mathbf{0} \\
\mathbf{R}(0)=\mathbf{R}_{\left\{0,0, \frac{75 \pi}{180}\right\}}, \boldsymbol{\Omega}(0)=\mathbf{0}
\end{array}\right.
$$

- Convincing behaviour of the proposed controller: Simulation results are reported in Figs. 2-6. Fig. 2 illustrates the convergence in 3-dimensional space of the actual trajectory to the reference trajectory. The time evolutions of the vehicle's position $\boldsymbol{\xi}_{\tilde{\xi}}$ against the reference position $\boldsymbol{\xi}_{r}$, of the position error $\tilde{\boldsymbol{\xi}}$, of the vehicle's orientation

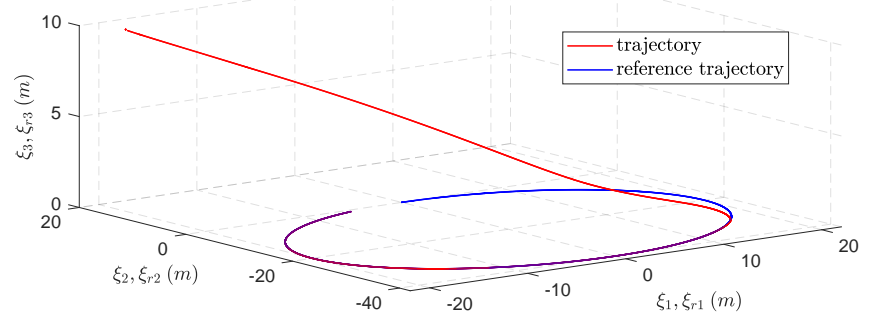

Fig. 2. (Proposed controller) Actual and reference trajectories
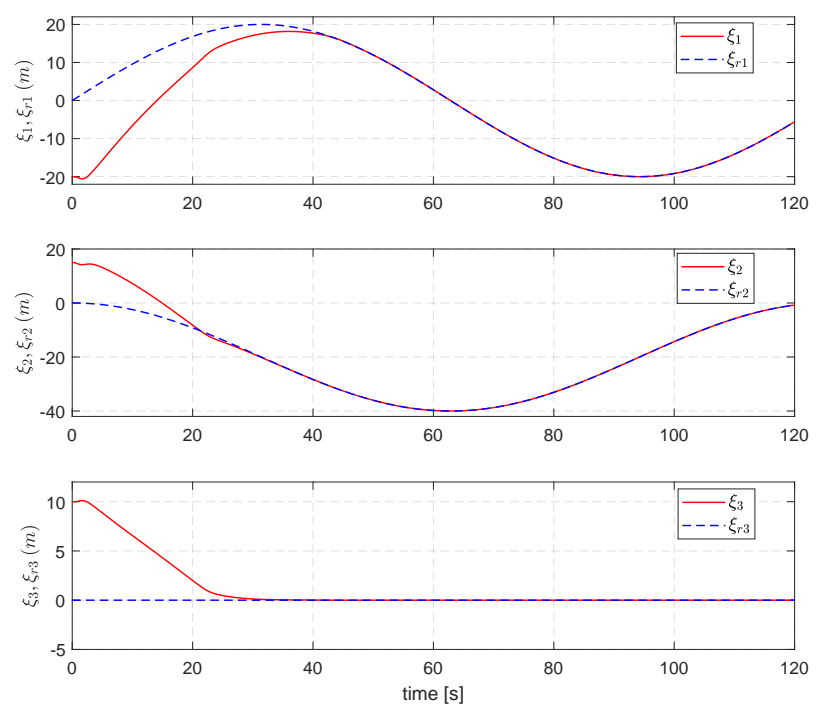

Fig. 3. (Proposed controller) Vehicle's position and reference position components v.s. time.

(represented by Euler angles), and of the applied force and torque control inputs are respectively shown in Figs. 3, 4, 5, and 6. From both Figs. 3 and 4 one observes that despite large initial errors and significant external disturbances, the vehicle's position converges quickly to the reference one without much oscillations and overshoots. Saturation in force (resp. yaw torque) control input occurred during the first $23[s]$ (resp. $3[s]$ ) as shown in Fig. 6 marginally affects the smooth convergence of the position error to zero. One can also observe from Fig. 5 that during the transient period the Euler roll angle always remains small (i.e. less than 5 degrees) and all the three Euler angles do not exhibit much oscillations. We find that the overall performance of the proposed controller is quite satisfactory.

- Oscillating behaviour of a simpler controller: In order to illustrate the need of introducing the augmented variable $\mathbf{u}$ and the associated control variable $\boldsymbol{\Omega}_{u}$ in the proposed control approach, simulation results using the simpler outerloop controller (21) are reported next. Similarly to the proposed outer-loop controller, the expression of $\boldsymbol{\Omega}_{d}$ given in (21) is slightly modified by adding a term $\lambda \mathbf{e}_{1}$, with $\lambda$ given in (39), so that the roll angle is also regulated near zero. The inner-loop controller is the same as the one used previously. All the gains and parameters involved in this controller are chosen identically to those used for the previously reported controller (i.e. given in (39)). Let us call this controller "simple controller" for distinguishing 


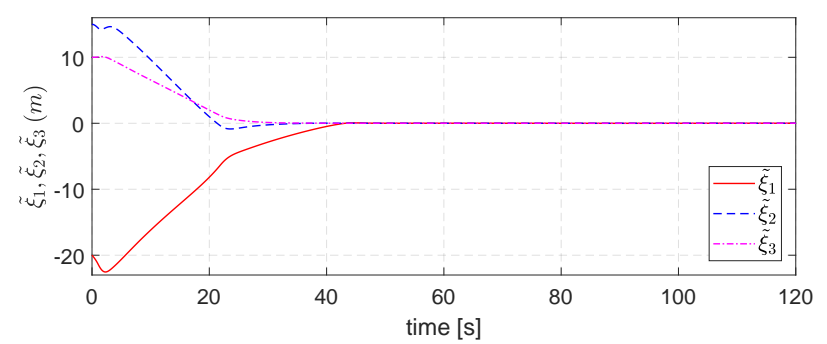

Fig. 4. (Proposed controller) Position tracking error v.s. time.

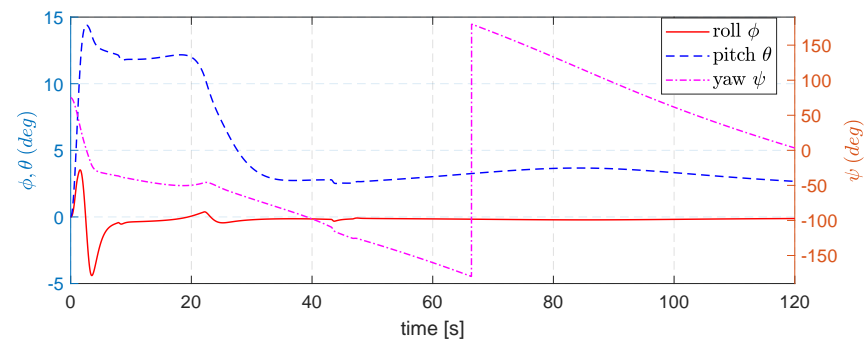

Fig. 5. (Proposed controller) Vehicle's orientation represented by Euler roll, pitch, yaw angles v.s. time.
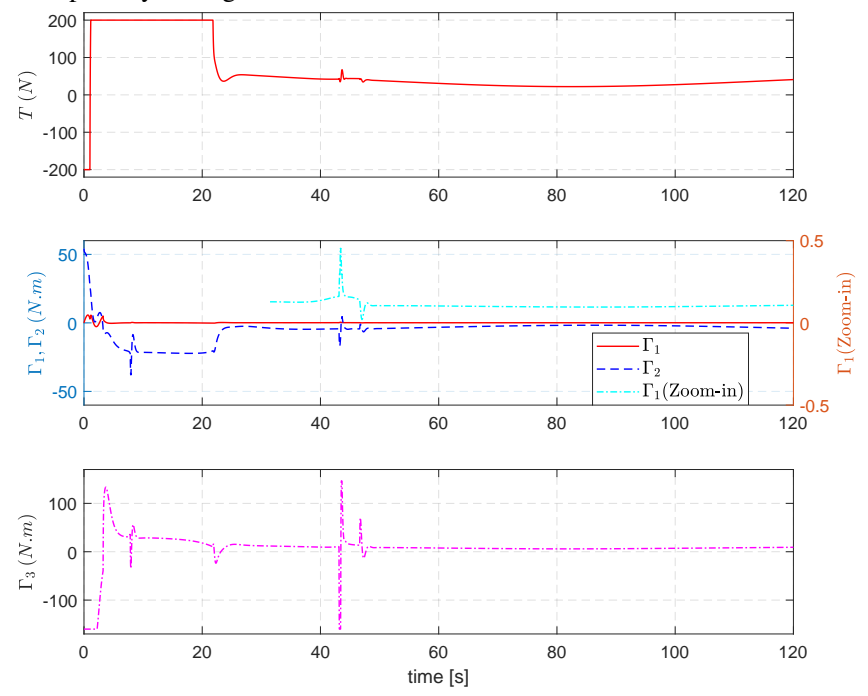

Fig. 6. (Proposed controller) Control force and torque inputs v.s. time.

with the proposed one. One observes that although the position error still converges to zero (see Fig. 7) the vehicle's orientation exhibits much more oscillations during the first 10 seconds of the transient phase (see Fig. 8) in contrast with the smooth behaviour of the proposed controller as shown in Fig. 5. This justifies the need of explicitly controlling the orientation rather than leaving the latter guided by the closed-loop system's zero dynamics.

\section{CONCLUSION}

A novel nonlinear control approach for slender-body axisymmetric underactuated underwater vehicles is proposed. Added-mass effects and dissipative hydrodynamic force are carefully taken into account via various adaptations, resulting in a modified apparent force no longer depending on the vehicle's orientation which is a good conditioning for control design. The proposed controller is also complemented with an integral correction term to enhance its robustness. Convincing simulation results conducted on a realistic model

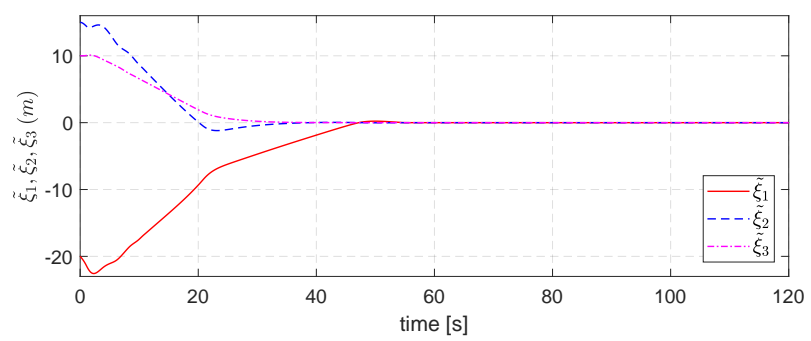

Fig. 7. (Simple controller) Position tracking error v.s. time.

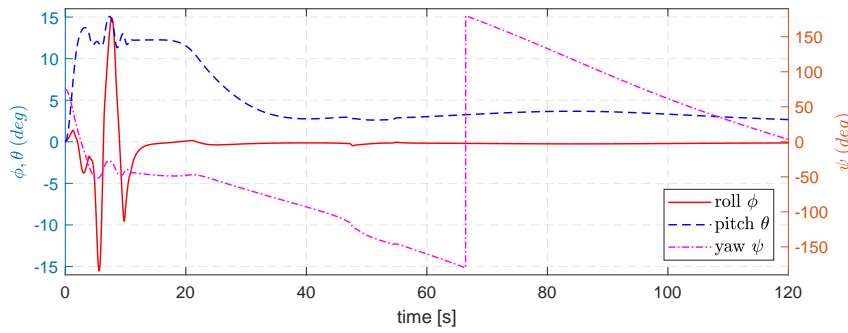

Fig. 8. (Simple controller) Vehicle's orientation represented by Euler roll, pitch, yaw angles v.s. time.

of a quasi-axisymmetric underwater vehicle illustrate the performance and robustness of the proposed controller.

Acknowledgement: This work was supported by the French projects: ANR ASTRID CONGRE (ANR-18-ASTR-0006), FUI GreenExplorer, ANR EQUIPEX ROBOTEX (ANR-10EQPX-44-01).

\section{REFERENCES}

[1] A. P. Aguiar and J. P. Hespanha. Trajectory-tracking and pathfollowing of underactuated autonomous vehicles with parametric modeling uncertainty. IEEE Transactions on Automatic Control, 52(8):1362-1379, 2007.

[2] A. P. Aguiar and A. M. Pascoal. Dynamic positioning and way-point tracking of underactuated AUVs in the presence of ocean currents. International Journal of Control, 80(7):1092-1108, 2007.

[3] T. I. Fossen. Guidance and control of ocean vehicles. John Wiley and Sons, New York, 1994.

[4] M.-D. Hua, T. Hamel, P. Morin, and C. Samson. A control approach for thrust-propelled underactuated vehicles and its application to VTOL drones. IEEE Transactions on Automatic Control, 54(8):1837$1853,2009$.

[5] M.-D. Hua and C. Samson. Time sub-optimal nonlinear PI and PID controllers applied to longitudinal headway car control. International Journal of Control, 84(10):1717-1728, 2011.

[6] J.-M. Kai, T. Hamel, and C. Samson. A nonlinear approach to the control of a disc-shaped aircraft. In IEEE Conference on Decision and Control (CDC), pages 2750-2755, 2017.

[7] H. Lamb. Hydrodynamics. Cambridge Univ. Press, 1932.

[8] N. E. Leonard. Stability of a bottom-heavy underwater vehicle. Automatica, 33(3):331-246, 1997.

[9] A. Micaelli and C. Samson. Trajectory tracking for unicycle-type and two-steering-wheels mobile robots. Technical Report 2097, INRIA, 1993.

[10] D. Pucci, T. Hamel, P. Morin, and C. Samson. Nonlinear feedback control of axisymmetric aerial vehicles. Automatica, 53:72-78, 2015.

[11] J. E. Refsnes, A. J. Sorensen, and K. Y. Pettersen. Model-based output feedback control of slender-body underactuated AUVs: theory and experiments. IEEE Transactions on Control Systems Technology, 16(5):930-946, 2008.

[12] F. Repoulias and E. Papadopoulos. Planar trajectory planning and tracking control design for underactuated AUVs. Ocean Engineering, 34(11):1650-1667, 2007.

[13] D. A. Smallwood and L. Whitcomb. Model-based dynamic positioning of underwater robotic vehicles: Theory and experiments. IEEE Journal of Oceanic Engineering, 29(1):169-186, 2004. 